Kragujevac Journal of Mathematics

Volume 38(1) (2014), Pages 95-103.

\title{
COUNTING RELATIONS FOR GENERAL ZAGREB INDICES
}

\author{
G. BRITTO ANTONY XAVIER ${ }^{1}$, E. SURESH ${ }^{2}$, AND I. GUTMAN ${ }^{3}$
}

\begin{abstract}
The first and second general Zagreb indices of a graph $G$, with vertex set $V$ and edge set $E$, are defined as $M_{1}^{k}=\sum_{v \in V} d(u)^{k}$ and $M_{2}^{k}=\sum_{u v \in E}[d(u) d(v)]^{k}$, where $d(v)$ is the degree of the vertex $v$ of $G$. We present combinatorial identities, relating $M_{1}^{k}$ and $M_{2}^{k}$ with counts of various subgraphs contained in the graph $G$.
\end{abstract}

\section{INTRODUCTION}

Throughout this paper we consider finite undirected and simple graphs. Let $G$ be such a graph, with vertex set $V=V(G)$ and edge set $E=E(G)$. Thus, $G$ has $|V|$ vertices and $|E|$ edges. The degree (= number of first neighbors) of a vertex $u \in V(G)$ is denoted by $d_{G}(u)$ or simply by $d(u)$ when the underlying graph is evident. For $u, v \in V(G)$, the distance (= length of a shortest path) between $u$ and $v$ is denoted by $d(u, v)$. The diameter of the graph $G$ is the greatest distance between its two vertices, and is denoted by $\operatorname{diam}(G)$.

In what follows, the path, star, and cycle with $n$ vertices will be denoted by $P_{n}$, $K_{1, n-1}$, and $C_{n}$ respectively.

Let $G$ and $H$ be graphs. We denote by $\sigma_{G}(H)$ the number of distinct subgraphs of the graph $G$ which are isomorphic to $H$. In particular, the graph $G$ has $\sigma_{G}\left(P_{1}\right)$ vertices, $\sigma_{G}\left(P_{2}\right)$ edges, and $\sigma_{G}\left(C_{3}\right)$ triangles. In addition,

$$
\sigma_{G}\left(K_{1, m}\right)=\sum_{v \in V(G)}\left(\begin{array}{c}
d(v) \\
m
\end{array}\right) .
$$

Let $\alpha$ and $\beta$ be positive integers. The double star $D_{\alpha, \beta}$ is the tree on $\alpha+\beta+2$ vertices, obtained from the path $P_{2}$, by attaching $\alpha$ pendent vertices to its one vertex, and $\beta$ pendent vertices to its other vertex.

Key words and phrases. Degree (of vertex), Zagreb index, General Zagreb index. 2010 Mathematics Subject Classification. Primary: 05C07, Secondary: 05C90.

Received: April 21, 2014. 
It is easy to see that for $\alpha \neq \beta$,

$$
\sigma_{G}\left(D_{\alpha, \beta}\right)=\sum_{u v \in E(G)}\left[\left(\begin{array}{c}
d(u)-1 \\
\alpha
\end{array}\right)\left(\begin{array}{c}
d(v)-1 \\
\beta
\end{array}\right)+\left(\begin{array}{c}
d(u)-1 \\
\beta
\end{array}\right)\left(\begin{array}{c}
d(v)-1 \\
\alpha
\end{array}\right)\right]
$$

whereas

$$
\sigma_{G}\left(D_{\alpha, \alpha}\right)=\sum_{u v \in E(G)}\left(\begin{array}{c}
d(u)-1 \\
\alpha
\end{array}\right)\left(\begin{array}{c}
d(v)-1 \\
\alpha
\end{array}\right) .
$$

\section{ZAGREB AND GENERAL ZAGREB INDICES}

In the 1970s, Trinajstić and one of the present authors introduced two vertexdegree-based graph invariants $[18,17]$, that eventually were named the first and the second Zagreb indices [1]. These are defined as

$$
M_{1}(G)=\sum_{v \in V(G)} d(v)^{2}
$$

and

$$
M_{2}(G)=\sum_{u v \in E(G)} d(u) d(v) .
$$

The first Zagreb index satisfies the important identity

$$
M_{1}(G)=\sum_{u v \in E(G)}[d(u)+d(v)]
$$

which was recognized in the mathematico-chemical literature only around 2010 [8]. Notice that this relation is the special case of Eq. (2.1) for $n=1$, which in turn is a special case of the recently discovered formula $(2.2)$ for $f(v):=d(v)^{n+1}[9]$

$$
\begin{aligned}
\sum_{v \in V(G)} d(v)^{n+1} & =\sum_{u v \in E(G)}\left[d(u)^{n}+d(v)^{n}\right] \\
\sum_{v \in V(G)} f(v) & =\sum_{u v \in E(G)}\left[\frac{f(u)}{d(u)}+\frac{f(v)}{d(v)}\right] .
\end{aligned}
$$

In [21], Li and Zheng introduced the first general Zagreb index as

$$
M_{1}^{k}(G)=\sum_{v \in V(G)} d(v)^{k}
$$

where $k \in \mathbb{R}$. Obviously $M_{1}^{0}(G)=|V|, M_{1}^{1}(G)=2|E|$, and $M_{1}^{2}(G) \equiv M_{1}(G)$. It is worth noting that a term identical to $M_{1}^{3}(G)$ was considered already in [18].

In analogy with Eq. (2.3), we now define the second general Zagreb index as

$$
M_{2}^{k}(G)=\sum_{u v \in E(G)}[d(u) d(v)]^{k}
$$

where $k \in \mathbb{R}$. Obviously $M_{2}^{0}(G)=|E|$ and $M_{2}^{1}(G) \equiv M_{2}(G)$. 
In 1992, Székely, Clark, and Entringer [26] proved that for every integer $k \geq 1$, $M_{1}^{k}(G) \leq\left[M_{1}^{1 / k}(G)\right]^{k}$. De Caen [7] proved that for every simple graph $G$ $M_{1}(G) \leq|V|\left(\frac{2|E|}{|V|-1}+|V|-2\right)$. This bound was generalized to hypergraphs by Bey [2] and sharpened by Das [4, 5]. De Caen's inequality was also used by Li and Pan[20] to provide an upper bound on the largest eigenvalue of the Laplacian of a graph.

In [3], Cioaba proved that for any positive number $k$,

$$
M_{1}^{k+1}(G) \leq \frac{2|E|}{|V|}\left[M_{1}^{k}(G)+(|V|-1)\left(\Delta^{k}-\delta^{k}\right)\right]-\frac{\Delta^{k}-\delta^{k}}{|V|} M_{1}(G)
$$

where $\Delta$ and $\delta$ are, respectively, the maximum and minimum degrees of the graph $G$.

Surveys on Zagreb indices are found in $[25,14,6,12,16,13]$. In $[29,28,22]$ various properties and relations of the first general Zagreb index are discussed. In addition to this, Edelberg et al. [11] elaborated the concept of subtree counting, using the characteristic polynomial of the distance matrix of a tree. Motivated by these results, we have proved a few combinatorial counting identities pertaining to the first and second general Zagreb indices.

\section{Main Results}

Theorem 3.1. Let $G$ be a simple graph. Then

$$
\begin{aligned}
M_{1}^{2}(G)= & 2 \sigma_{G}\left(K_{1,2}\right)+2|E| \\
M_{1}^{3}(G)= & 3 ! \sigma_{G}\left(K_{1,3}\right)+6 \sigma_{G}\left(K_{1,2}\right)+2|E| \\
M_{1}^{4}(G)= & 4 ! \sigma_{G}\left(K_{1,4}\right)+36 \sigma_{G}\left(K_{1,3}\right)+14 \sigma_{G}\left(K_{1,2}\right)+2|E| \\
M_{1}^{5}(G)= & 5 ! \sigma_{G}\left(K_{1,5}\right)+240 \sigma_{G}\left(K_{1,4}\right)+150 \sigma_{G}\left(K_{1,3}\right) \\
& +30 \sigma_{G}\left(K_{1,2}\right)+2|E| \\
M_{1}^{6}(G)= & 6 ! \sigma_{G}\left(K_{1,6}\right)+1800 \sigma_{G}\left(K_{1,5}\right)+1560 \sigma_{G}\left(K_{1,4}\right) \\
& +540 \sigma_{G}\left(K_{1,3}\right)+62 \sigma_{G}\left(K_{1,2}\right)+2|E| \\
M_{1}^{7}(G)= & ! \sigma_{G}\left(K_{1,7}\right)+15120 \sigma_{G}\left(K_{1,6}\right)+16800 \sigma_{G}\left(K_{1,5}\right) \\
& +8400 \sigma_{G}\left(K_{1,4}\right)+1806 \sigma_{G}\left(K_{1,3}\right)+126 \sigma_{G}\left(K_{1,2}\right)+2|E| \\
M_{1}^{8}(G)= & 8 ! \sigma_{G}\left(K_{1,8}\right)+141120 \sigma_{G}\left(K_{1,7}\right)+191520 \sigma_{G}\left(K_{1,6}\right) \\
& +126000 \sigma_{G}\left(K_{1,5}\right)+40824 \sigma_{G}\left(K_{1,4}\right)+5796 \sigma_{G}\left(K_{1,3}\right) \\
& +254 \sigma_{G}\left(K_{1,2}\right)+2|E| \\
& +1905120 \sigma_{G}\left(K_{1,6}\right)+834120 \sigma_{G}\left(K_{1,5}\right)+186480 \sigma_{G}\left(K_{1,4}\right) \\
& +10150 \sigma_{G}\left(K_{1,3}\right)+510 \sigma_{G}\left(K_{1,2}\right)+2|E|
\end{aligned}
$$




$$
\begin{aligned}
M_{1}^{10}(G)= & 10 ! \sigma_{G}\left(K_{1,10}\right)+16329600 \sigma_{G}\left(K_{1,9}\right)+30240000 \sigma_{G}\left(K_{1,8}\right) \\
& +29635200 \sigma_{G}\left(K_{1,7}\right)+16435440 \sigma_{G}\left(K_{1,6}\right) \\
& +5103000 \sigma_{G}\left(K_{1,5}\right)+818520 \sigma_{G}\left(K_{1,4}\right)+55980 \sigma_{G}\left(K_{1,3}\right) \\
& +1022 \sigma_{G}\left(K_{1,2}\right)+2|E| .
\end{aligned}
$$

Proof. Eq. (3.1) is a well known relation for the ordinary first Zagreb index [13]. The other equalities in Theorem 3.1 are obtained by consecutively applying Eqs. (1.1) and (2.3).

In particular, by applying Eqs. (1.1), (2.3), and (3.1) we get

$$
\begin{aligned}
\sigma_{G}\left(K_{1,3}\right) & =\sum_{v \in V(G)}\left(\begin{array}{c}
d(v) \\
3
\end{array}\right)=\frac{1}{3 !} \sum_{v \in V(G)} d(v)[d(v)-1][d(v)-2] \\
& =\frac{1}{3 !}\left[\sum_{v \in V(G)} d(v)^{3}-3 \sum_{v \in V(G)} d(v)^{2}+2 \sum_{v \in V(G)} d(v)\right] \\
& =\frac{1}{3 !}\left[M_{1}^{3}(G)-3 M_{1}^{2}(G)+2 M_{1}^{1}(G)\right] \\
& =\frac{1}{3 !}\left[M_{1}^{3}(G)-3\left(2 \sigma_{G}\left(K_{1,2}\right)+2|E|\right)+2(2|E|)\right]
\end{aligned}
$$

from which Eq. (3.2) straightforwardly follows. Next, using Eqs. (1.1), (2.3), (3.1), and (3.2), we get

$$
\begin{aligned}
\sigma_{G}\left(K_{1,4}\right)= & \sum_{v \in V(G)}\left(\begin{array}{c}
d(v) \\
4
\end{array}\right)=\frac{1}{4 !} \sum_{v \in V(G)} d(v)[d(v)-1][d(v)-2][d(v)-3] \\
= & \frac{1}{4 !}\left[\sum_{v \in V(G)} d(v)^{4}-6 \sum_{v \in V(G)} d(v)^{3}+11 \sum_{v \in V(G)} d(v)^{2}-6 \sum_{v \in V(G)} d(v)\right] \\
= & \frac{1}{4 !}\left[M_{1}^{4}(G)-6 M_{1}^{3}(G)+11 M_{1}^{2}(G)-6 M_{1}^{1}(G)\right] \\
= & \frac{1}{3 !}\left[M_{1}^{3}(G)-6\left(3 ! \sigma_{G}\left(K_{1,3}\right)+6 \sigma_{G}\left(K_{1,2}\right)+2|E|\right)\right. \\
& \left.+11\left(2 \sigma_{G}\left(K_{1,2}\right)+2|E|\right)-6(2|E|)\right]
\end{aligned}
$$

from which Eq. (3.3) straightforwardly follows. The remaining equalities are obtained recursively in a fully analogous manner. 
Corollary 3.1. Let $G$ be a simple graph. Then,

$$
\begin{aligned}
M_{1}^{3}(G)= & 3 ! \sigma_{G}\left(K_{1,3}\right)+3 M_{1}^{2}(G)-4|E| \\
M_{1}^{4}(G)= & 4 ! \sigma_{G}\left(K_{1,4}\right)+6 M_{1}^{3}(G)-11 M_{1}^{2}(G)+12|E| \\
M_{1}^{5}(G)= & 5 ! \sigma_{G}\left(K_{1,5}\right)+10 M_{1}^{4}(G)-35 M_{1}^{3}(G)+50 M_{1}^{2}(G)-48|E| \\
M_{1}^{6}(G)= & 6 ! \sigma_{G}\left(K_{1,6}\right)+15 M_{1}^{5}(G)-85 M_{1}^{4}(G)+225 M_{1}^{3}(G) \\
& -274 M_{1}^{2}(G)+240|E| \\
M_{1}^{7}(G)= & 7 ! \sigma_{G}\left(K_{1,7}\right)+21 M_{1}^{6}(G)-175 M_{1}^{5}(G)+735 M_{1}^{4}(G)-1624 M_{1}^{3}(G) \\
& +1764 M_{1}^{2}(G)-1440|E| \\
M_{1}^{8}(G)= & 8 ! \sigma_{G}\left(K_{1,8}\right)+28 M_{1}^{7}(G)-322 M_{1}^{6}(G)+1960 M_{1}^{5}(G) \\
& -6769 M_{1}^{4}(G)+13132 M_{1}^{3}(G)-13068 M_{1}^{2}(G)+10080|E| \\
M_{1}^{9}(G)= & ! \sigma_{G}\left(K_{1,9}\right)+36 M_{1}^{8}(G)-546 M_{1}^{7}(G)+4536 M_{1}^{6}(G)-22449 M_{1}^{5}(G) \\
& +67284 M_{1}^{4}(G)-118124 M_{1}^{3}(G)+109584 M_{1}^{2}(G)-80640|E| \\
M_{1}^{10}(G)= & 10 ! \sigma_{G}\left(K_{1,10}\right)+45 M_{1}^{9}(G)-870 M_{1}^{8}(G)+9450 M_{1}^{7}(G) \\
& -63273 M_{1}^{6}(G)+269325 M_{1}^{5}(G)-723680 M_{1}^{4}(G)+1172700 M_{1}^{3}(G) \\
& -1026576 M_{1}^{2}(G)+725760|E| .
\end{aligned}
$$

Theorem 3.2. Let $T$ be a tree and $m$ an integer such that $2<m \leq \operatorname{diam}(T)$. Then

$$
\sigma_{T}\left(P_{m+1}\right)=\sum_{d(u, v)=m-2}\left[d_{T}(u)-1\right]\left[d_{T}(v)-1\right] .
$$

Proof. We prove (3.4) by induction on the number of vertices of $T$.

If $|V|=4,|E|=3$, then $T$ must be either $P_{4}$ or $K_{1,3}$ and hence $T$ satisfies (3.4).

For a subtree $T_{1}=T-v$, where $v$ is a pendent vertex of $T$, assume that (3.4) holds for $T_{1}$. Therefore the induction hypothesis is

$$
\sigma_{T_{1}}\left(P_{m+1}\right)=\sum_{d(x, y)=m-2}\left[d_{T_{1}}(x)-1\right]\left[d_{T_{1}}(y)-1\right] .
$$

Since $u v \notin E\left(T_{1}\right)$ and $u v \in E(T), \sigma_{T_{1}}\left(P_{m+1}\right)$ can be expressed as

$$
\begin{aligned}
\sigma_{T_{1}}\left(P_{m+1}\right)= & {\left[d_{T_{1}}(u)-1\right]\left[d_{T_{1}}\left(u_{1}\right)-1\right]+\left[d_{T_{1}}(u)-1\right]\left[d_{T_{1}}\left(u_{2}\right)-1\right] } \\
& +\cdots+\left[d_{T_{1}}(u)-1\right]\left[d_{T_{1}}\left(u_{k}\right)-1\right]+\text { other terms in } T_{1}
\end{aligned}
$$

where $d\left(u, u_{i}\right)=m-2, i=1,2,3, \ldots, k$.

Since for all $x \in V\left(T_{1}\right) \backslash\{u\}, \quad d_{T}(x)=d_{T_{1}}(x)$ whereas $d_{T}(u)=d_{T_{1}}(u)+1$, we have

$$
\begin{aligned}
\sigma_{T_{1}}\left(P_{m+1}\right)= & {\left[\left(d_{T}(u)-1\right)-1\right]\left[d_{T}\left(u_{1}\right)-1\right]+\left[\left(d_{T}(u)-1\right)-1\right]\left[d_{T}\left(u_{2}\right)-1\right] } \\
& +\cdots+\left[\left(d_{T}(u)-1\right)-1\right]\left[\left(d_{T}\left(u_{k}\right)-1\right)-1\right]+\text { other terms in } T .
\end{aligned}
$$


Therefore, $\sigma_{T_{1}}\left(P_{m+1}\right)=\sigma_{T}\left(P_{m+1}\right)-\sum_{i=1}^{k}\left[d_{T}\left(u_{i}\right)-1\right]$ and

$$
\begin{aligned}
\sum_{i=1}^{k}\left[d_{T}\left(u_{i}\right)-1\right]= & \sum_{\substack{i=1 \\
d\left(u, u_{i}\right)=m-2}}^{k}\left[\left(d_{T_{1}}(u)+1\right)-1\right]\left[d_{T_{1}}\left(u_{i}\right)-1\right] \\
& -\sum_{\substack{i=1 \\
d\left(u, u_{i}\right)=m-2}}^{k}\left[d_{T_{1}}(u)-1\right]\left[d_{T_{1}}\left(u_{i}\right)-1\right] .
\end{aligned}
$$

This implies,

$$
\begin{aligned}
\sigma_{T}\left(P_{m+1}\right)= & \sigma_{T_{1}}\left(P_{m+1}\right)+\sum_{\substack{i=1 \\
d\left(u, u_{i}\right)=m-2}}^{k}\left[\left(d_{T_{1}}(u)+1\right)-1\right]\left[d_{T_{1}}\left(u_{i}\right)-1\right] \\
& -\sum_{\substack{i=1 \\
d\left(u, u_{i}\right)=m-2}}^{k}\left[d_{T_{1}}(u)-1\right]\left[d_{T_{1}}\left(u_{i}\right)-1\right]
\end{aligned}
$$

From (3.5) and (3.6) we complete the proof of Theorem 3.2.

Remark 3.1. The special case of formula (3.4) for $m=3$, i.e.,

$$
\sigma_{T}\left(P_{4}\right)=\sum_{u v \in E(T)}\left[d_{T}(u)-1\right]\left[d_{T}(v)-1\right]
$$

was first established in 2009 [10], and later also in [23, 19, 27]. Its extension to triangle-containing graphs is also known [24]. Within these researches, a relation equivalent to Eq. (3.7), namely

$$
M_{2}(G)=M_{1}(G)+\sigma_{G}\left(P_{4}\right)-|E|
$$

was also reported. For more details on this matter see $[15,16,13]$.

Corollary 3.2. Let $T$ be a tree and $m$ an integer such that $1<m \leq \operatorname{diam}(T)$. Then $\sigma_{T}\left(P_{m+1}\right)=\frac{1}{2} \sum_{d(u, v)=m-1}\left[\left(d_{T}(u)-1\right)+\left(d_{T}(v)-1\right)\right]$.

Corollary 3.3. Let $G$ be a $C_{i}$-free graph $(2<i \leq m)$. Then

$$
\begin{aligned}
\sigma_{G}\left(P_{m+1}\right) & =\sum_{d(u, v)=m-2}[d(u)-1][d(v)-1] \\
& =\frac{1}{2} \sum_{d(u, v)=m-1}[(d(u)-1)+(d(v)-1)] .
\end{aligned}
$$

Eq. (3.8) can be rewritten as

$$
M_{2}^{1}(G)=\sigma_{G}\left(D_{1,1}\right)+M_{1}^{2}(G)-|E| .
$$

In the next theorem we show that analogous relations can be found also in the case of higher-order second general Zagreb indices, $M_{2}^{k}(G), k \geq 2$. 
Theorem 3.3. Let $G$ be a triangle-free graph. Then

$$
\begin{aligned}
M_{2}^{2}(G)= & 4 \sigma_{G}\left(D_{2,2}\right)+6 \sigma_{G}\left(D_{1,2}\right)+9 M_{2}^{1}(G)+M_{1}^{3}(G) \\
& -9 M_{1}^{2}(G)+8|E| \\
M_{2}^{3}(G)= & 36 \sigma_{G}\left(D_{3,3}\right)+144 \sigma_{G}\left(D_{2,2}\right)+72 \sigma_{G}\left(D_{2,3}\right) \\
& +42 \sigma_{G}\left(D_{1,3}\right)+84 \sigma_{G}\left(D_{1,2}\right)+49 M_{2}^{1}(G) \\
& +M_{1}^{4}(G)-49 M_{1}^{2}(G)+48|E| .
\end{aligned}
$$

Proof. Using Eq. (1.2), we have

$$
\begin{aligned}
\sigma_{G}\left(D_{1,2}\right)= & \sum_{u v \in E(G)}\left[\left(\begin{array}{c}
d(u)-1 \\
1
\end{array}\right)\left(\begin{array}{c}
d(v)-1 \\
2
\end{array}\right)+\left(\begin{array}{c}
d(u)-1 \\
2
\end{array}\right)\left(\begin{array}{c}
d(v)-1 \\
1
\end{array}\right)\right] \\
= & \frac{1}{2} \sum_{u v \in E(G)}\left[\left[d(u)^{2} d(v)+d(v)^{2} d(u)\right]\right. \\
& \left.-6 d(u) d(v)-\left[d(u)^{2}+d(v)^{2}\right]+5[d(u)+d(v)]-4\right] \\
= & \frac{1}{2} \sum_{u v \in E(G)}\left[d(u)^{2} d(v)+d(v)^{2} d(u)\right] \\
& -\frac{1}{2}\left(6 M_{2}^{1}(G)+M_{1}^{3}(G)-5 M_{1}^{2}(G)+4|E|\right)
\end{aligned}
$$

i.e.,

$$
\begin{aligned}
\sum_{u v \in E(G)}\left[d(u)^{2} d(v)+d(v)^{2} d(u)\right]= & 2 \sigma_{G}\left(D_{1,2}\right)+6 M_{2}^{1}(G) \\
& +M_{1}^{3}(G)-5 M_{1}^{2}(G)+4|E|
\end{aligned}
$$

where we used Eq. (2.1) and the definitions (2.3) and (2.4) of the first and second general Zagreb indices.

Using Eq. (1.3) and similar arguments, we obtain

$$
\begin{aligned}
\sigma_{G}\left(D_{2,2}\right)= & \sum_{u v \in E(G)}\left(\begin{array}{c}
d(u)-1 \\
2
\end{array}\right)\left(\begin{array}{c}
d(v)-1 \\
2
\end{array}\right) \\
= & \frac{1}{4} \sum_{u v \in E(G)}\left[d(u)^{2} d(v)^{2}+9 d(u) d(v)+2\left[d(u)^{2}+d(v)^{2}\right]\right. \\
& \left.-6[d(u)+d(v)]+4-3\left[d(u)^{2} d(v)+d(v)^{2} d(u)\right]\right] \\
= & \frac{1}{4}\left(M_{2}^{2}(G)+9 M_{2}^{1}(G)+2 M_{1}^{3}(G)-6 M_{1}^{2}(G)+4|E|\right) \\
& -\frac{3}{4} \sum_{u v \in E(G)}\left[d(u)^{2} d(v)+d(v)^{2} d(u)\right]
\end{aligned}
$$


which combined with Eq. (3.12) yields

$$
\sigma_{G}\left(D_{2,2}\right)=\frac{1}{4}\left[M_{2}^{2}(G)-9 M_{2}^{1}(G)-M_{1}^{3}(G)+9 M_{1}^{2}(G)-8|E|-6 \sigma_{G}\left(D_{1,2}\right)\right]
$$

which is equivalent to Eq. (3.10).

The proof of Eq. (3.11) is analogous, yet somewhat more lengthy.

Identities of the same kind as Eqs. (3.10) and (3.11) could be deduced also for second general Zagreb indices $M_{2}^{k}(G), k \geq 4$.

Substituting Eqs. (3.1)-(3.3) and (3.9) back into the relations in Theorem 3.3, we obtain the following.

Corollary 3.4. Let $G$ be a triangle-free graph. Then

$$
\begin{aligned}
M_{2}^{2}(G)= & 4 \sigma_{G}\left(D_{2,2}\right)+6 \sigma_{G}\left(D_{1,2}\right)+6 \sigma_{G}\left(K_{1,3}\right)+9 \sigma_{G}\left(P_{4}\right)+6 \sigma_{G}\left(P_{3}\right)+|E| \\
M_{2}^{3}(G)= & 36 \sigma_{G}\left(D_{3,3}\right)+144 \sigma_{G}\left(D_{2,2}\right)+72 \sigma_{G}\left(D_{2,3}\right)+42 \sigma_{G}\left(D_{1,3}\right)+84 \sigma_{G}\left(D_{1,2}\right) \\
& +24 \sigma_{G}\left(K_{1,4}\right)+36 \sigma_{G}\left(K_{1,3}\right)+49 \sigma_{G}\left(P_{4}\right)+14 \sigma_{G}\left(P_{3}\right)+|E| .
\end{aligned}
$$

\section{REFERENCES}

[1] A. T. Balaban, I. Motoc, D. Bonchev and O. Mekenyan, Topological indices for structure-activity correlations, Topics Curr. Chem. 114 (1983), 21-55.

[2] C. Bey, An upper bound on the sum of squares of degrees in a hypergraph, Discrete Math. 269 (2003), 259-263.

[3] S. M. Cioabă, Sums of powers of the degrees of a graph, Discrete Math. 41 (2006), 1959-1964.

[4] K. C. Das, Sharp bounds for the sum of the squares of the degrees of a graph, Kragujevac J. Math. 25 (2003), 31-49.

[5] K. C. Das, Maximizing the sum of the squares of the degrees of a graph, Discrete Math. 285 (2004), 57-66.

[6] K. C. Das and I. Gutman, Some properties of the second Zagreb index, MATCH Commun. Math. Comput. Chem. 52 (2004), 103-112.

[7] D. de Caen, An upper bound on the sum of squares of degrees in a graph, Discrete Math. 285 (2004), 57-66.

[8] T. Došlić, B. Furtula, A. Graovac, I. Gutman, S. Moradi and Z. Yarahmadi, On vertex-degreebased molecular structure descriptors, MATCH Commun. Math. Comput. Chem. 66 (2011), 613-626.

[9] T. Došlić, T. Réti and D. Vukičević, On the vertex degree indices of connected graphs, Chem. Phys. Lett. 512 (2011), 283-286.

[10] W. Du, X. Li and Y. Shi, Algorithms and extremal problem on Wiener polarity index, MATCH Commun. Math. Comput. Chem. 62 (2009), 235-244.

[11] M. Edelberg, M. R. Garey and R. L. Graham, On the distance matrix of a tree, Discrete Math. 14 (1976), 23-39.

[12] I. Gutman, Degree-based topological indices, Croat. Chem. Acta 86 (2013), 351-361.

[13] I. Gutman, On the origin of two degree-based topological indices, Bull. Acad. Serbe Sci. Arts. (Cl. Sci. Math.), in press.

[14] I. Gutman and K. C. Das, The first Zagreb indices 30 years after, MATCH Commun. Math. Comput. Chem. 50 (2004), 83-92. 
[15] I. Gutman, B. Furtula and C. Elphick, Three new/old vertex-degree-based topological indices, MATCH Commun. Math. Comput. Chem. in press.

[16] I. Gutman and T. Réti, Zagreb group indices and beyond, Int. J. Chem. Model. 6 (2014), 000-000.

[17] I. Gutman, B. Ruščić, N. Trinajstić and C. F. Wilcox, Graph theory and molecular orbitals. XII. Acyclic polyenes, J. Chem. Phys. 62 (1975), 3399-3405.

[18] I. Gutman and N. Trinajstić, Graph theory and molecular orbitals. Total $\pi$-electron energy of alternant hydrocarbons, Chem. Phys. Lett. 17 (1972), 535-538.

[19] H. Hou and B. Liu, The maximum Wiener polarity index of unicyclic graphs, Appl. Math. Comput. 218 (2012), 10149-10157.

[20] J. S. Li and Y. L. Pan, De Caen's inequality and bounds on the largest Laplacian eigenvalue of a graph, Linear Algebra Appl. 328 (2001), 153-160.

[21] X. Li and J. Zheng, A unified approach to the extremal trees for different indices, MATCH Commun. Math. Comput. Chem. 54 (2005), 195-208.

[22] M. Liu and B. Liu Some properties of the first general Zagreb index, Australas. J. Comb. 47 (2010), 285-294.

[23] M. Liu and B. Liu, On the Wiener polarity index, MATCH Commun. Math. Comput. Chem. 66 (2011), 293-304.

[24] M. Milošević, T. Réti and D. Stevanović, On the constant difference of Zagreb indices, MATCH Commun. Math. Comput. Chem. 68 (2012), 157-168.

[25] S. Nikolić, G. Kovačević and A. Miličević, The Zagreb indices 30 years after, Croat. Chem. Acta 76 (2003), 113-124.

[26] L. A. Székely, L. H. Clark and R. C. Entriger, An inequality for degree sequences, Discrete Math. 103 (1992), 293-300.

[27] D. Vukičević and T. Pisanski, On the extremal values of the ratios of the number of paths, Ars. Math. Contemp. 3 (2010), 215-235.

[28] S. Zhang, W. Wang and T. C. E. Cheng, Bicyclic graphs with the first three smallest and largest values of the first general Zagreb index, MATCH Commun. Math. Comput. Chem. 55 (2006), 579-592.

[29] H. Zhang and S. Zhang, Unicyclic graphs with the first three smallest and largest first general Zagreb index, MATCH Commun. Math. Comput. Chem. 55 (2006), 427-438.

\footnotetext{
${ }^{1}$ Department of Mathematics,

SaCred Heart College,

TiRupatTuR-635601, TAMil NADU,

INDIA

E-mail address: shcbritto@yahoo.in

${ }^{2}$ Department of Mathematics, Velammal Engineering College, Surapet, Chennai-600066, Tamil Nadu, INDIA

E-mail address: sureshkako@gmail.com

${ }^{3}$ FACUlty of Science,

University of Kragujevac,

P. O. Box 60, 34000 Kragujevac,

SERBIA

E-mail address: gutman@kg.ac.rs
} 